\title{
Caractérisation expérimentale du phénomène d'auto-cicatrisation des fissures dans les bétons
}

\author{
Sébastien Granger
}

\section{R\&DO - Institut de Recherche en Génie Civil et Mécanique (GeM) - UMR CNRS 6183, Ecole Centrale de Nantes - 1, rue de la Noë - 44321 Nantes Cedex 3}

\begin{abstract}
RESUME. Le phénomène d'auto-cicatrisation des fissures dans les bétons est étudié par le biais de diverses techniques expérimentales. Un programme basé sur le vieillissement d'éprouvettes pré-fissurées en flexion 3 points et la caractérisation de leur comportement mécanique, est ainsi mis en place. Les résultats mécaniques, à savoir une reprise rapide de rigidité globale et une légère amélioration de la capacité portante, sont corrélés aux analyses microscopiques réalisées sur les fissures cicatrisées. Parallèlement aux essais mécaniques, des techniques de contrôle non destructif sont développées pour analyser le phénomène. L'émission acoustique permet ainsi de mettre en évidence la micro-fissuration des produits néoformés dans la fissure, ainsi que de caractériser les mécanismes de fissuration des éprouvettes cicatrisées. Les premiers résultats obtenus par miroirs à retournement temporel pour le suivi in vivo du phénomène, sont également présentés.
\end{abstract}

MOTS-CLÉS : auto-cicatrisation, contrôle non destructif, analyses microscopiques

\begin{abstract}
Self healing of cracks in concrete is investigated through different experimental techniques. An experimental program, based on the ageing of pre-cracked specimens and the characterization of their mechanical behaviour, is thus performed. The mechanical results, a fast recovery of initial stiffness and a slow improvement of flexural resistance, are correlated with microscopic investigations carried out on the healed cracks. In addition to the mechanical tests, non destructive testing techniques are developed to analyze the phenomenon. Acoustic emission highlights the micro-cracking of newly formed crystals in the cracks, and characterizes the cracking processes of healed specimens. The first results of time reversal mirrors experiments, for the following in live of the phenomenon, are also reported.
\end{abstract}

KEYWORDS : self healing, non destructive testing, microscopic analysis

\section{INTRODUCTION}

Pour beaucoup de structures en béton en fin de vie, ou encore en service, la question de la durabilité du matériau apparaît comme une donnée essentielle, pour assurer le fonctionnement en service et la sécurité des ouvrages. La présence de fissures est un des facteurs importants influant sur la durabilité du matériau béton, notamment en termes de résistance ou propriétés de transfert. L'autocicatrisation des fissures est un phénomène physico-chimique ayant des répercussions positives sur ces problèmes de durabilité. Ce phénomène apparaît en présence d'eau entres les deux lèvres d'une fissure, et l'accumulation des cristaux issus des réactions chimiques qui sont la cause du phénomène, forme un pont susceptible de rétablir la continuité. La présence de composés capables de réagir avec l'eau est donc essentielle, et c'est le ciment, hydraté ou non, qui est l'autre élément essentiel au phénomène. Deux grandes hypothèses sont avancées pour expliquer le phénomène (Neville, 2002) : la précipitation de cristaux colmatants (notamment la calcite) et l'hydratation du ciment anhydre présent dans la microstructure du béton durci. Dans les recherches antérieures, le phénomène a été 
principalement mis en évidence par des essais de perméabilité à l'eau, expliquant la diminution voire l'annulation du débit de fuite au travers d'un échantillon de béton fissuré, par l'occurrence de la cicatrisation (Hearn, 1997 ; Edvardsen, 1999 ; Reinhardt et al, 2003). Parallèlement à cela, peu d'études ont été menées sur l'apport mécanique du phénomène. La reprise de fréquence de résonance d'éprouvettes initialement fissurées, puis ensuite cicatrisées a néanmoins été mise en évidence (Jacobsen et al., 1996 ; Pimienta et al., 2004).

Ce travail de recherche s'inscrit donc dans la volonté d'apporter de nouveaux éléments pour la compréhension d'un phénomène encore mal connu. L'étude a été lancée dans le contexte de développement des bétons fibrés à ultra hautes performances (BFUP). L'objectif principal est de quantifier l'apport mécanique du phénomène, ce qui permettrait de justifier la prise en compte du comportement post-pic des modèles de comportement associés aux BFUP. Un programme expérimental est ainsi élaboré, comprenant le vieillissement d'éprouvettes pré-fissurées, suivi de la caractérisation de leur comportement mécanique. Le développement de techniques de contrôle non destructif (émission acoustique, miroirs à retournement temporel) pour l'auscultation du béton est associé à ces essais mécaniques.

\section{CARACTERISATION MECANIQUE DU PHENOMENE DE CICATRISATION}

\subsection{DESCRIPTION DU MATERIAU ET DES EPROUVETTES}

Le programme expérimental est mené sur la matrice d'un béton fibré à ultra hautes performances (BFUP). La cicatrisation apparaissant exclusivement dans la pâte de ciment, et pour des raisons liées à l'utilisation en parallèle d'un système d'émission acoustique, il a été décidé de supprimer les fibres et d'étudier uniquement la matrice du matériau. Le rapport Eau/Ciment est de l'ordre de 0,2, ce qui implique une quantité de ciment anhydre de l'ordre de 50 à $60 \%$ au sein de la microstructure du béton durci (Loukili et al., 1998). Le matériau est donc considéré comme modèle, dans la mesure où cette forte proportion de ciment anhydre est un facteur très favorable d'apparition du phénomène de cicatrisation. Les éprouvettes utilisées sont prismatiques, entaillées, et de dimensions 50x100x500 $\mathrm{mm}^{3}$. Elles ont subi un traitement thermique assurant leur stabilité chimique (réactions d'hydratation stoppées).

\subsection{PRINCIPE DES ESSAIS MECANIQUES}

Les essais mécaniques comportent 3 phases :

- une phase de pré-fissuration en flexion 3 points (voir figure 1) permettant une fissuration contrôlée de toutes les éprouvettes. Une largeur de fissure unique de l'ordre de $10 \mu \mathrm{m}$ est visée.

- le vieillissement des éprouvettes dans l'eau (immersion complète à $20^{\circ} \mathrm{C}$ ) ou dans l'air (à $20^{\circ} \mathrm{C}$ et $50 \% \mathrm{HR}$ ), pour des périodes allant de 1 à 20 semaines

- la caractérisation mécanique des éprouvettes vieillies en flexion 3 points 


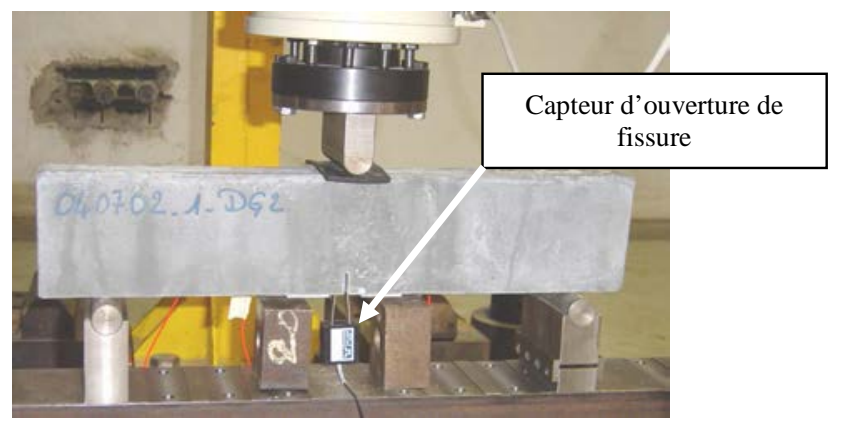

Figure 1 : Configuration des essais mécaniques.

\subsection{MISE EN EVIDENCE MECANIQUE DU PHENOMENE}

La figure 2 présente les résultats mécaniques moyens, obtenus sur 3 éprouvettes, lors de la phase de rechargement des éprouvettes vieillies, pour 2 périodes différentes : 10 et 20 semaines. On observe ainsi que le comportement des éprouvettes vieillies dans l'air est exactement le même pour les deux périodes de vieillissement. Des essais complémentaires de référence (Granger et al., 2006) consistant en la fissuration d'éprouvettes et leur rechargement immédiat, montrent que ce comportement mécanique observé pour les éprouvettes conservées dans l'air est typiquement le rechargement classique d'éprouvettes après fissuration. L'air n'a donc aucune influence sur les propriétés mécaniques des éprouvettes de béton fissurées. Au contraire, il y a une nette évolution du comportement mécanique en fonction du temps de vieillissement dans l'eau.
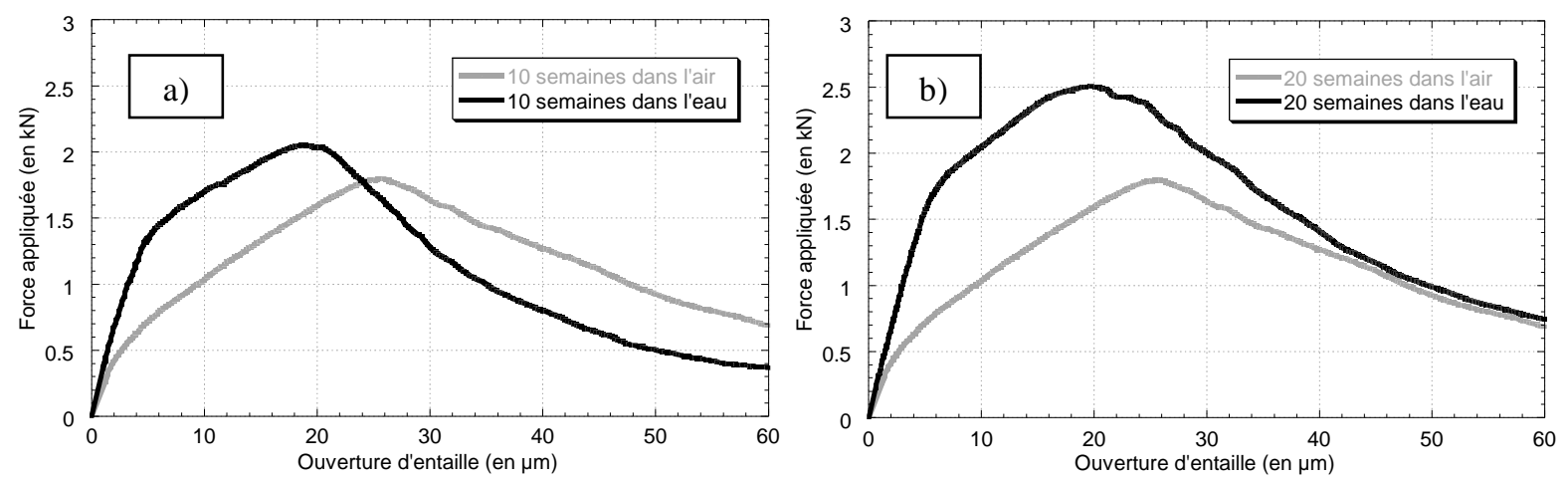

Figure 2 : Comportement mécanique moyen lors de la phase de rechargement pour les éprouvettes vieillies dans l'air et dans l'eau ; a) 10 semaines, b) 20 semaines.

La principale différence de comportement mécanique entre éprouvettes fissurées conservées dans l'air et dans l'eau, réside dans une reprise de rigidité globale, et dans une légère reprise de capacité portante, croissantes avec le temps. Ces résultats mécaniques sont imputés à la cicatrisation de la fissure initialement formée. Des éprouvettes non fissurées conservées dans l'eau pendant plusieurs semaines, puis fissurées et rechargées immédiatement, confirment qu'il n'y a pas d'amélioration des propriétés mécaniques par cure humide, et que ce comportement typique est bien dû à la cicatrisation (Granger et al., 2006).

La figure 3 montre l'évolution, en fonction du temps de vieillissement, du rapport entre la raideur globale des éprouvettes cicatrisées et celle des éprouvettes fissurées. Après 10 semaines de vieillissement, on a pratiquement atteint le rapport correspondant à la raideur initiale, c'est à dire celles des éprouvettes saines. Cette reprise de rigidité peut donc être directement reliée à la 
précipitation de nouveaux cristaux dans la fissure, en particulier de nouveaux C-S-H, dont la raideur est sans doute peu éloignée de celles des hydrates formés lors de l'hydratation primaire.

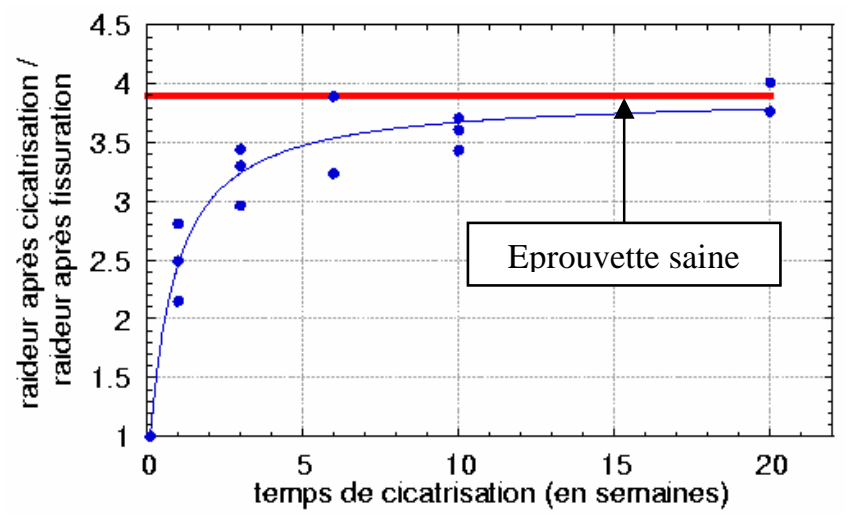

Figure 3 : Evolution du rapport raideur globale après cicatrisation/raideur après endommagement et sans cicatrisation, en fonction du temps de vieillissement.

Le comportement mécanique des éprouvettes conservées dans l'eau met également en évidence une augmentation de la capacité portante, par rapport au cas du vieillissement dans l'air. La figure 4 représente l'évolution de la force appliquée au pic, pour les éprouvettes cicatrisées, en comparaison avec la force moyenne pour les éprouvettes non cicatrisées représentée par le trait continu.

On constate qu'il y a une évolution assez lente de la capacité portante des éprouvettes avec le temps de cicatrisation. Néanmoins, on n'atteint pas la résistance des éprouvettes saines. Ce résultat peut être expliqué par le développement progressif des propriétés mécaniques des nouveaux cristaux formés dans la fissure, ou alors celle de l'interface qui s'est créée entre cristaux nouvellement formés et ceux issus de l'hydratation primaire du ciment.

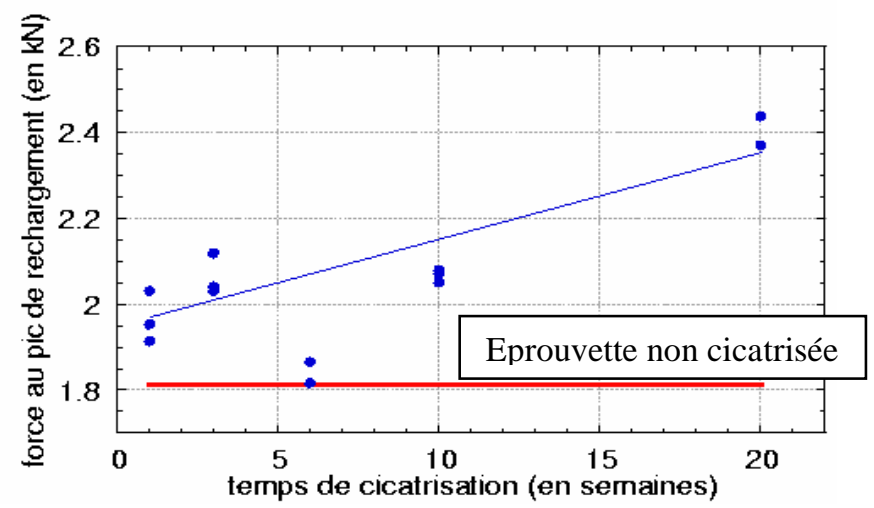

Figure 4: Evolution de la force au pic de rechargement en fonction du temps de cicatrisation.

\section{OBSERVATIONS MICROSCOPIQUES}

Afin d'étayer les hypothèses formulées quant au comportement mécanique des éprouvettes cicatrisées, des observations microscopiques sont menées sur les surfaces de fissure après rupture. Elles sont réalisées par microscopie électronique à balayage et complétées par des analyses qualitatives par spectrométrie à sélection d'énergie, pour déterminer la nature des produits 
précipitants. La figure 5 représente une fractographie typique du béton, respectivement pour une surface de fissure non cicatrisée et pour une surface de fissure cicatrisée, à un grossissement x10000.
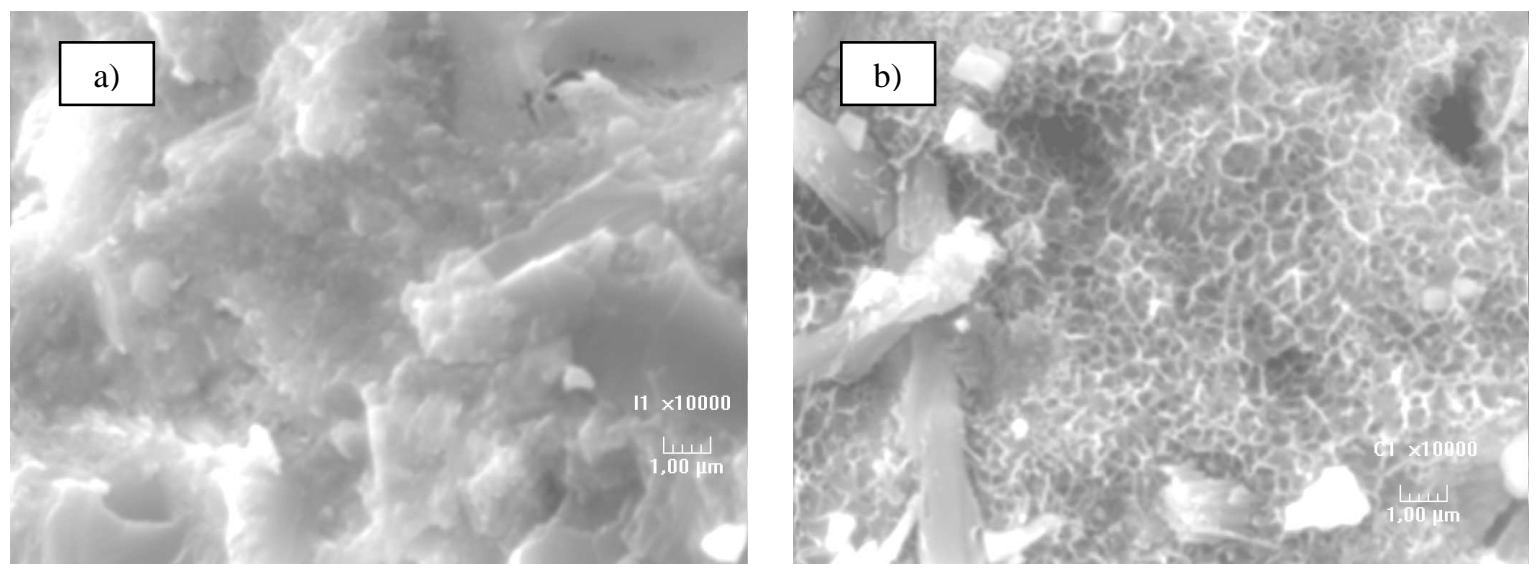

Figure 5 : Fractographies du béton étudié ; a) fissure non cicatrisée, b) fissure cicatrisée.

Les observations sur la surface de fissure non cicatrisée (figure 5a) mettent en évidence la microstructure du béton étudié. On est en présence d'une matrice très dense, avec des amas de C-S-H formant une structure amorphe. Les observations réalisées sur les surfaces de fissures cicatrisées (figure 5b) sont radicalement différentes. En effet, on observe des amas de cristaux en pelotes d'aspect beaucoup moins dense que le gel très compact observé sur les fissures brutes. Les résultats des analyses par spectrométrie à sélection d'énergie confirment ainsi qu'on est en présence typique de C$\mathrm{S}-\mathrm{H}$ alvéolaires. On peut par ailleurs remarquer sur la fractographie des petites particules cubiques blanches, de dimensions de l'ordre du $\mu \mathrm{m}$, dont l'analyse par spectrométrie révèle qu'elles pourraient s'apparenter à des cristaux de calcite. On ne peut clairement l'affirmer puisqu'il existe différentes formes du carbonate de calcium.

Ces observations microscopiques sont ainsi en accord avec les hypothèses formulées pour le comportement mécanique des éprouvettes cicatrisées. La cristallisation de nouveaux C-S-H sur pratiquement toute la surface de la fissure cicatrisée va clairement dans le sens de la reprise de rigidité globale de l'éprouvette. Concernant la reprise de résistance en flexion, on constate bien que la différence de texture des amas de C-S-H, très dense dans le cas de l'hydratation primaire, et de structure alvéolaire dans le cas des cristaux formés par cicatrisation, permet de comprendre pourquoi il est difficile de recréer le matériau localement et de lui redonner sa résistance complète.

\section{CARACTERISATION PAR DES TECHNIQUES NON DESTRUCTIVES}

\subsection{EMISSION ACOUSTIQUE}

Pendant les différentes phases des essais mécaniques, une instrumentation émission acoustique (EA) est mise en place. Elle a pour but d'obtenir des informations sur les processus de fissuration des éprouvettes cicatrisées, et des informations sur les propriétés mécaniques des cristaux formés. Quatre transducteurs piézoélectriques (avec une fréquence de résonance de $150 \mathrm{kHz}$ ) sont placés sur les éprouvettes juste au dessus de l'entaille, afin d'entourer la zone de micro-fissuration. Les transducteurs formant une maille de localisation rectangulaire de 140x65 mm² (voir figure 6). Ils sont 
placés sur une face de l'éprouvette, et couplés au matériau avec une colle silicone (Granger et al, 2006).

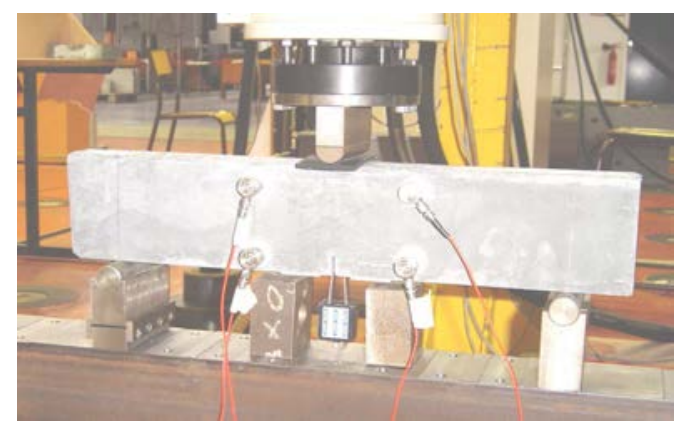

Figure 6 : Instrumentation émission acoustique - Maille de localisation de $140 \times 65 \mathrm{~mm}^{2}$.

Des cartes de micro-fissuration sont ainsi obtenues lors de la phase de rechargement des éprouvettes, cicatrisées ou non. La figure 7 représente des cartes typiques obtenues en phase pré-pic pour des éprouvettes respectivement cicatrisées et non cicatrisées, pendant 10 semaines. Les évènements détectés ont été filtrés en énergie, et seuls les évènements d'énergie supérieure à 1000 atto Joules sont conservés. On s'affranchit ainsi de tous les signaux peu énergétiques détectés dans la zone en dehors de la macro-fissure (Otsuka, 2000). Cette figure met ainsi clairement en évidence la microfissuration des produits formés dans la fissure pour le cas éprouvette cicatrisée, alors que l'on a une réouverture de la fissure sans détection acoustique pour l'éprouvette non cicatrisée.
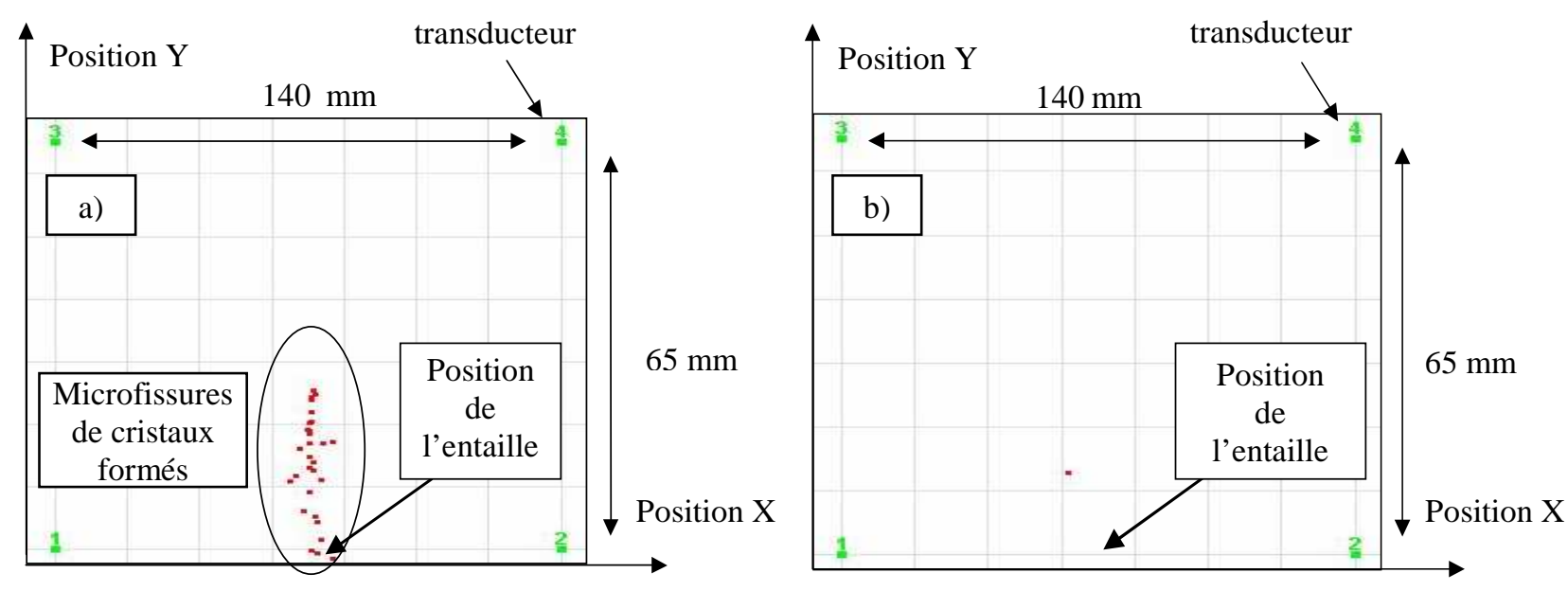

Figure 7 : Cartes de microfissuration, pendant la phase de rechargement (phase pré-pic jusqu'à 18 $\mu m)$ pour des éprouvettes vieillies 10 semaines ; a) dans l'eau, b) dans l'air.

L'analyse plus précise de ces cartes de localisation peut être menée en terme d'énergie des microfissures détectées. La figure 8 présente les cartes de micro-fissuration en énergie pour une éprouvette cicatrisée et pour une éprouvette non cicatrisée, à $90 \%$ en phase post pic. A ce stade, on a ainsi quasiment endommagé tous les cristaux qui se sont formés dans la fissure. 


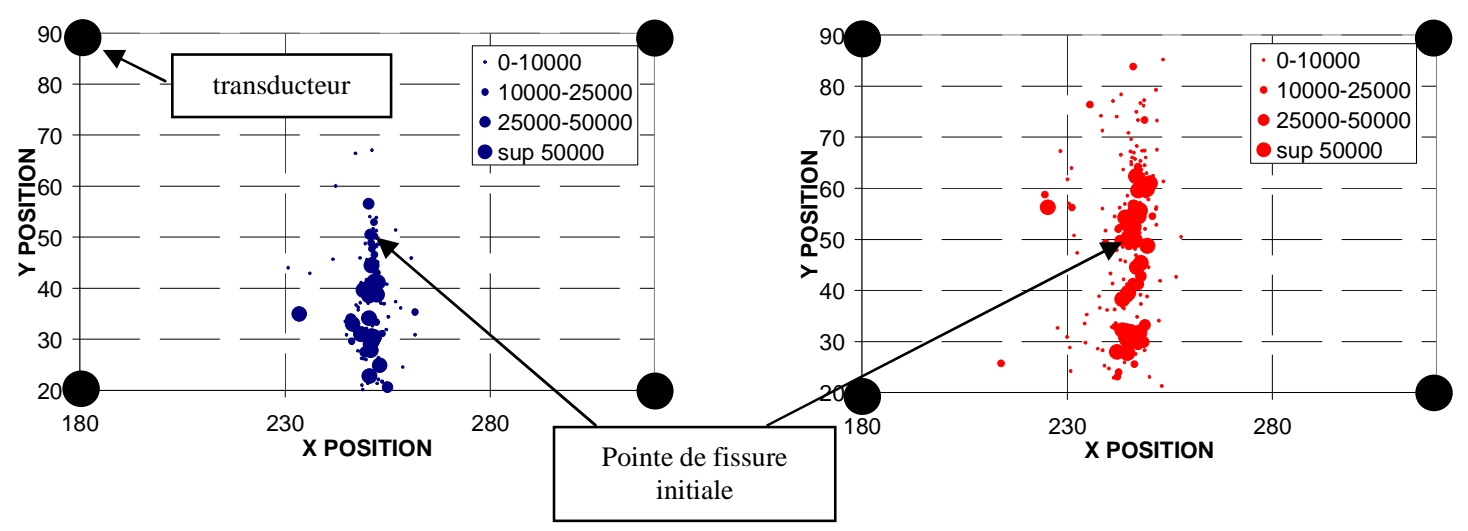

Figure 8: Cartes de localisation en énergie à $90 \%$ de la force au pic en phase post pic; a) éprouvette cicatrisée, b) éprouvette non cicatrisée

Ainsi, presque $100 \%$ de l'energie dissipée se trouve dans la fissure pré-existante dans le cas où il y a cicatrisation, alors que ce poucentage est de moins de $50 \%$ dans le cas où il n'y a pas cicatrisation (les détections étant dues à des frottements ou des réouvertures de micro-fissures). On met ainsi en évidence les 2 régimes de fissuration des éprouvettes cicatrisées, à savoir la micro-fissuration des produits néo-formés dans un premier temps suivie de la poursuite de la propagation de la fissure préexistante dans un second temps. Si nous sommons maintenant la totalité de l'énergie dissipée à ce stade, on peut s'apercevoir qu'elle est environ $50 \%$ plus faible dans le cas de l'éprouvette cicatrisée. Cette énergie correspondant à l'energie libérée par la fissuration des produits néoformés, alors qu'elle est liée à la fissuration de matériau sain dans le cas de non cicatrisation, on est ainsi en droit de penser que les produits de cicatrisation ont des propriétés mécaniques différentes de celles du matériau sain.

\subsection{MIROIR A RETOURNEMENT TEMPOREL}

La technique de retournement temporel réside dans la possibilité de focaliser des ondes ultrasonores dans des milieux complexes (Fink, 1999). En pratique, un réseau de capteurs, appelé miroir à retournement temporel, est capable, après avoir enregistré le signal provenant d'une source acoustique, de le ré-émettre retourné dans le temps, et ainsi de le faire focaliser au niveau de la source.

L'idée d'expérience associée à la cicatrisation des fissures est ainsi de suivre périodiquement la focalisation de signaux au niveau d'une pointe de fissure cicatrisant, et d'être ainsi capable de suivre le phénomène in vivo. La technique n'ayant jamais été employée pour de tels matériaux, et présentant des difficultés de mise en œuvre conséquentes, des étapes préliminaires de focalisation dans le béton ont été réalisées afin de valider la focalisation d'ondes ultrasonores au sein du béton. Un essai de flexion 3 points instrumenté est ensuite réalisé pour étudier l'évolution de la focalisation avec le développement d'endommagement. Un transducteur placé dans la zone de propagation de la fissure émet un pulse, détecté par un miroir composé de 4 capteurs. Le signal enregistré sur ce miroir est retourné puis renvoyé dans le matériau, et la focalisation au point émetteur est étudiée en fonction de l'avancement de l'endommagement (voir figure 9). On s'aperçoit ainsi de l'évolution du spectre fréquentiel du signal focalisé en fonction de l'évolution de l'endommagement. L'intérêt est maintenant de savoir mener le même type d'essais dans le cas de la cicatrisation, et ainsi être capable de retrouver la focalisation initiale, avec l'avancement du phénomène. 

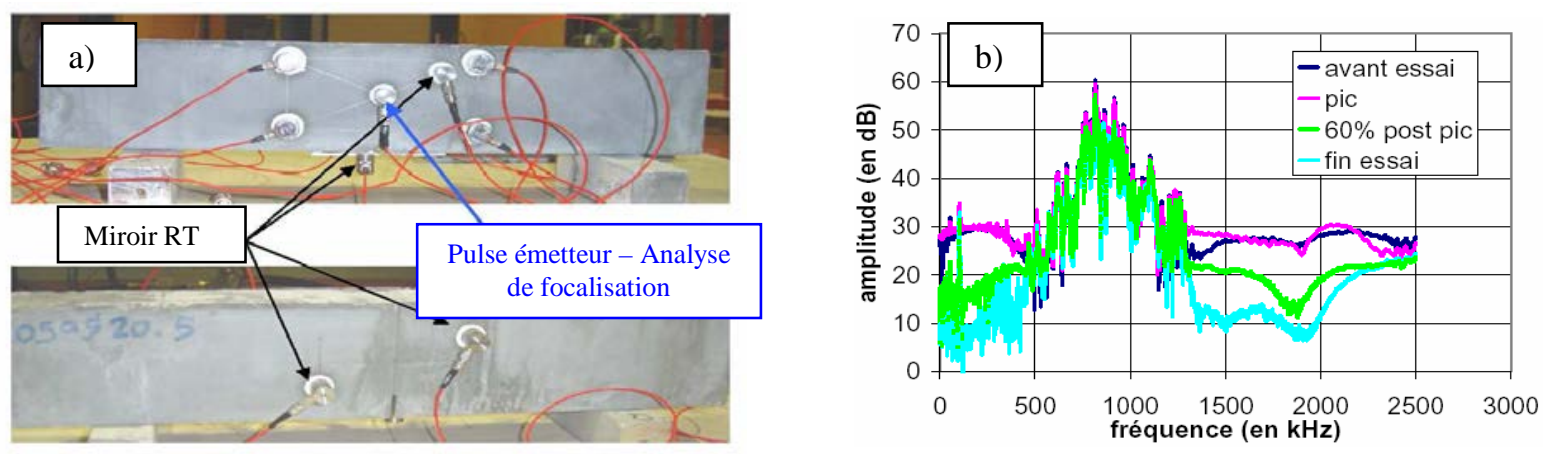

Figure 9 : Evolution de la focalisation par retournement temporel en fonction de l'endommagement ;

a) instrumentation, b) spectre fréquentiel du signal focalisé.

\section{CONCLUSION}

Le programme expérimental développé pour caractériser l'auto-cicatrisation a permis de mettre en évidence l'apport du phénomène sur le plan mécanique. Cet apport se manifeste par une reprise rapide de rigidité des éprouvettes endommagées et une légère amélioration de leur capacité portante. Ces résultats ont été imputés à la précipitation de nouveaux hydrates dans la fissure, ce qui est confirmé par les observations microscopiques et les analyses par spectrométrie à sélection d'énergie effectuées. En paralléle des essais mécaniques, le développement des techniques de contrôle non destructif basées sur l'émission acoustique et les miroirs à retournement temporel ont permis de tirer des informations qualitatives sur les processus de fissuration des éprouvettes cicatrisées, ainsi que d'établir les premières étapes d'instrumentations originales pour le suivi du phénomène en direct.

\section{BIBLIOGRAPHIE}

Neville A. (2002) «Autogenous healing - A concrete miracle? » Concrete International, Novembre 2002 , p. $76-82$

Edvardsen C. (1999) «Water permeability and autogenous healing of cracks in concrete » ACI Material Journal, vol. 96, nº , p. 448-454

Hearn N. (1997) «Self-healing property of concrete - Experimental evidence» Materials and Structures, vol. 30, p. 404-411

Reinhardt H.W., Joos M. (2003) «Permeability and self-healing of cracked concrete as a function of temperature and crack width » Cement and Concrete Research, vol. 33, n 7, p. 981-985

Jacobsen S., Sellevold E. (1996) «Self-healing of high strength concrete after deterioration by freeze/thaw »Cement and Concrete Research, vol.26, ${ }^{\circ} 1$, p. 55-62

Pimienta P., Chanvillard G. (2004) « Retention of the mechanical performances of Ductal ${ }^{\circledR}$ specimens kept in various aggressive environments » Fib - Symposium 2004, Avignon

Loukili A., Richard P., Lamirault J. (1998) «A study on delayed deformations of an ultra high strength cementious material » ACI SP179-59, Recent Advances in Concrete Technology, vol. 179

Granger S., A.Loukili, G.Pijaudier-Cabot, G.Chanvillard (2006) «Caractérisation expérimentale du phénomène d'auto-cicatrisation des fissures sur un BUHP » REGC (à paraître)

Otsuka K., Date H. (2000) «Fracture process zone in concrete tension specimen » Cement and Concrete Research, vol. 65, n²-3, p. 111-131

Fink M. (1999) «Time reversed acoustics » Scientific American, November 1999, p.67-73 